\title{
A Percepção dos Servidores Públicos da Escola de Administração Tributária quanto ao uso do GECAP
}

\author{
The Perception of the Servers Public School tax Administration \\ Regarding the use of GECAP
}

\section{Cleber Soares de Brito}

Mestrando no Programa de Pós-Graduação Gestão em Organizações Aprendentes - UFPB; Gerente Operacional de Educação Continuada da Escola de Administração Tributária da Secretaria de Estado da Receita da Paraíba - João Pessoa - PB - Brasil. E-mail: clebersoares33@ig.com.br

\section{Maria das Graças Vieira}

Professora da Universidade Federal de Pernambuco (UFPE) e do Programa de Pós-Graduação Gestão em Organizações Aprendentes - UFPB - Recife - PE - Brasil. E-mail: gracinhavieira@yahoo.com.br

\section{Resumo}

Com o dinamismo do novo cenário socioeconômico, definido pela influência das Tecnologias da Informação e Comunicação, provoca-se na sociedade mundial a transição de um modelo ultrapassado, fundamentado no indivíduo para outro, baseado no coletivo, cuja sociedade se coloca numa imensa rede de conexões que interagem entre si. Nesse sentido optar por investir no desenvolvimento de sistemas de informações que possam integrar os mais diversos setores da organização se torna uma necessidade crescente, pois agora são necessárias informações cada vez mais adequadas as suas atividades. Assim, optou-se em analisar os fatores que explicam o uso dos sistemas de informações. Tais fatores relacionam-se ao comportamento e à atitude dos indivíduos diante da tecnologia e do ajuste dela com a tarefa executada. De acordo com a Teoria da Ação Racional, da Teoria do Comportamento Planejado, do Modelo de Aceitação de Tecnologia e do Modelo de Adequação entre Tarefa e Tecnologia, chega-se à problemática deste estudo: de que forma os servidores da ESAT estão utilizando o GECAP?

Palavras-chave: Modelo de Administração Pública. Brasil. Angola.

\section{Abstract}

With the dynamism of the new socioeconomic scenario, defined by the influence of Information Technology and Communication, leads global society in the transition from an outdated model, based on the individual to another, based on the collective, where the company stands in a vast network of connections that interact. In this sense choose to invest in developing information systems that can integrate the most diverse sectors of the organization becomes an increasing need for now if you need more information appropriate to their activities. So it was decided to analyze the factors that explain the use of information systems. These factors relate to the behavior and attitude of individuals facing the technology and the adjustment of being performed. According to the Theory of Reasoned Action, Theory of Planned Behavior, the Technology Acceptance Model and Model Matching Task and Technology, one comes to the issue of this study: how the servers are using the ESAT GECAP?

Key words: Public Servant. Information Systems. Use. 


\section{INTRODUÇÃO}

Com o avanço tecnológico, as organizações em seus contextos complexos têm sofrido inúmeros problemas de gerenciamento de pessoas e informações. As empresas, sejam elas de direito público ou privado, estão inseridas em um ambiente tecnológico altamente competitivo e turbulento, que se transforma a todo instante, exigindo das empresas um sistema de informação ágil que acompanhe o ritmo das transformações. Segundo Neto (1999), a busca por competitividade, através de redução de custos e ganhos de produtividade, está fazendo com que as organizações procurem por inovações tecnológicas que permitam uma vantagem competitiva. Torres (1995) mostra que adequar tecnologia e objetivos estratégicos é um dos mais fortes fatores de competitividade. A competitividade de uma organização está relacionada ao meio ambiente na qual está inserida, dessa forma, ela deve considerar em suas estratégias as características desse ambiente. (PORTER, 1990)

O setor público, seja federal, estadual ou municipal, vem sendo influenciado pelas Tecnologias da Informação e Comunicação e tentando se adaptar a ela. $\mathrm{O}$ advento do governo na web e sua integração com ferramentas e métodos da tecnologia da informação (TI) fomentaram a criação de serviços oferecidos pela internet. O impulso inicial veio do setor privado, com o comércio eletrônico (e-commerce), mas atualmente as aplicações governamentais (e-government) já não são novidade. (BAZILLI, 2000)

Nesse cenário, o servidor público se constitui num verdadeiro estágio evolutivo do velho servidor, com a intervenção de nossa Constituição Federal de 1988, levando em consideração sua percepção de responsabilidade pública, a partir do surgimento dos concursos públicos e da ideia que se coloca de uma isonomia de oportunidades, resgatada pela nossa Constituição Federal de 1988. O foco desse novo servidor estaria baseado na legalidade, moralidade, publicidade, impessoalidade e na eficiência. (OLIVEIRA, 2008)

Algumas das atividades estatais (de cunho não monopolista e caráter competitivo, a exemplo dos serviços de saúde, educação, pesquisa, limpeza urbana, entre outros), passam a ser compartilhadas com a sociedade civil, para garantir a melhor prestação dos serviços públicos (BAZILI, 2000). Uma atividade estatal muito importante nesse sentido é a atividade da Secretaria de Estado da Receita (SER) (FISCO Estadual). Subordinado a SER tem-se a Escola de Administração Tributária (ESAT), tendo como objetivos permanentes o ensino, a pesquisa, a extensão e as ações educacionais de interesse da arrecadação, fiscalização $e$ tributação estadual, assim como a implementação de ações visando à conscientização do cidadão por meio do Programa de Educação Fiscal.

A utilização de novas tecnologias tem sido considerada vital para a sobrevivência da organização, principalmente a utilização de Sistemas de Informações (SI), que já está presente nas organizações, no cotidiano das pessoas, de empresas governamentais e não governamentais.

A análise do uso dos sistemas de informações pode ser verificada a partir do uso de modelos como a Teoria da Ação Racional (TRA), Teoria do Comportamento Planejado (TPB), o Modelo de Aceitação Tecnológico (TAM) e o modelo de Ajuste Tarefa Tecnologia (TTF), os quais se baseiam em diferentes aspectos e este artigo parte do princípio de que esses aspectos podem influenciar no uso do Gerenciador de Capacitação (GECAP), sistema de informações criado e editado na linguagem PHP, utilizado nas atividades diárias dos servidores públicos da Escola de Administração Tributária, localizada na cidade de João Pessoa.

Mesmo com toda a evolução tecnológica e os crescentes investimentos das organizações nessas tecnologias, o problema de subutilização de sistemas afeta vários negócios. Como bem observa Venkatesh (2000), investimentos bem-sucedidos em tecnologia podem levar a um substancial aumento de produtividade e, consequentemente, a um aumento de lucratividade. Por outro lado, problemas com os SI e seu uso podem implicar em resultados indesejados, como perdas financeiras e insatisfação dos funcionários.

Diante do exposto, faz-se necessário analisar o comportamento dos servidores da ESAT, no uso do GECAP já que desde a sua criação não foi realizada nenhuma análise das variáveis que estejam relacionados ao comportamento ou mesmo as atitudes dos indivíduos frente à tecnologia, e com o alinhamento dela com a tarefa executada. Assim, essa pesquisa se propõe responder à seguinte questão: de que forma os servidores da ESAT estão utilizando o GECAP? 


\section{Referencial Teórico}

As mudanças tecnológicas que se vive nas últimas décadas nos fazem refletir sobre o uso de sistemas de informação (SI) nas organizações, como também, do papel do servidor público nesse novo contexto. Assim, analisar o efeito desse SI na execução das atividades profissionais desse público tornou-se condição básica para a busca de uma maior eficiência das atividades públicas, resultando numa melhor prestação de serviço aos nossos clientes externos. Dessa forma otimizaram-se as atividades organizacionais públicas, na busca em se adequar a esses novos tempos, investindo-se na aquisição de software para que se acompanha essa evolução.

\subsection{Sistema de Informação}

A escolha, por parte dos gestores, na implantação de uma tecnologia da informação em uma organização é uma decisão que visa mudar o seu estado, com o intuito de aumentar a sua eficácia e eficiência. Os sistemas de informações (SI) podem ser definidos tecnicamente como um conjunto de componentes inter-relacionados, que coleta (ou recupera), processa, armazena e distribui informações destinadas a apoiar a tomada de decisões, à coordenação e ao controle, esses sistemas também auxiliam os gerentes e os colaboradores a analisar problemas da empresa. (LAUDON; LAUDON, 2010)

De acordo com esses autores, a coleta corresponde à entrada de dados brutos que caracterizam algum evento ocorrido. $\mathrm{O}$ processamento dos dados transforma-os em algo que tenha significado para a organização. $\mathrm{O}$ armazenamento permite que se possa ter acesso a estas informações no momento oportuno. Quanto a saída há uma transferência das informações processadas às pessoas que as utilizarão ou às atividades nas quais elas serão empregada Os sistemas de informações também requerem um feedback, que é uma resposta à ação adotada a determinados membros da organização para ajudá-los a avaliar ou corrigir o estágio de entrada. (LAUDON; LAUDON, 2010, p. 12)

A ESAT demanda de seus gestores ações e decisões estratégicas, cujos resultados podem ser avaliados por meio do Gerenciador de Capacitação (GECAP), sistema de informação implantado na ESAT. A partir dessa implantação podemos antecipar problemas, antever cenários, projetar novos rumos a serem seguidos. Por isso, não deve ater-se apenas ao presente, muito menos ao passado, mas sim imaginar o futuro que oriente as ações do presente.

O SI denominado Gerenciador de Capacitação (GECAP) foi criado e editado na linguagem PHP (um acrônimo recursivo para: Hypertext Preprocessor), trata-se de uma linguagem de script open source de uso geral, muito utilizada pelos programadores $e$ especialmente guarnecida para o desenvolvimento de aplicações Web embutível dentro do HTML. Tem como fonte de pesquisa um banco de dados próprio que é alimentado, administrado e editado pelo MySQL sistema de gerenciamento de banco de dados (SGBD), que utiliza a linguagem SQL (Structured Query Language) como interface. É atualmente um dos bancos de dados mais populares, com mais de 10 milhões de instalações pelo mundo.

Esse sistema é acessado a partir do portal da Secretaria Executiva da Receita, com um link de acesso em sua página principal. Utiliza-se nesse momento um método de comunicação interna chamada de intranet, espaço restrito para uso exclusivo dos servidores da SER para compartilhamento de informações restritas.

A intranet possibilita tudo o que a própria internet dispõe. Porém, a principal diferença entre ambas é que a intranet é restrita a certo público. Há restrição de acesso, por exemplo, por uma empresa, ou seja, todos os colaboradores da empresa podem acessar a intranet desde que possuam um nome de usuário e senha devidamente especificados pela coordenação da empresa. O acesso à intranet é feito em um servidor local em uma rede local chamada de LAN (rede de acesso local) instalada na própria empresa.

A partir da criação do Gerenciador de Capacitação (GECAP), tem-se um SI que além de disponibilizar informações, poderia também colaborar com outras funções importantes, que auxiliam as empresas a alcançarem seus objetivos a partir do uso da tecnologia, tais como: auxiliar na execução das tarefas, facilitando o controle e integrando processos; auxiliando nas decisões em todos os níveis, a partir da disponibilização da informação; ajudar a organização a se tornar mais eficiente em suas ações perante os nossos servidores públicos. 
O sucesso da adoção de TI está relacionado com o "saber escolher" e o "saber usar", o que pressupõe a assimilação de inovações tecnológicas, o alinhamento entre a TI $e$ as estratégias da organização, a elaboração de estratégias específicas para investimentos em TI, bem como as atitudes gerenciais e comportamentais voltadas para a inovação. (FERNANDES; ALVES, 1992, p. 72)

\subsection{Modelos de Sistemas de Informações}

Compreender o motivo de os usuários de um SI usarem ou rejeitarem determinados equipamentos de informática tornou-se uma das maiores inquietações dos estudiosos da área de TI (DAVIS; BAGOZZI; WARSHAW, 1989). Dessa forma, o foco desse artigo não estará presente no olhar técnico, mas na busca de compreender o comportamento de quem irá utilizá-la.

Destacam-se inicialmente as pesquisas sobre utilização da tecnologia baseada nos comportamentos $e$ atitudes dos indivíduos. Esse e o caso do modelo discutido por Ajzen e Fishbein (1975) denominado de Teoria da Ação Racional (Theory of Reasoned Action - TRA) (GOODHUE; THOMPSON, 1995). Esse modelo apresenta que o comportamento individual $e$ determinado pelas intenções de comportamento, que ocorrem em função da atitude do indivíduo definido como sentimentos positivos e negativos do mesmo. Para esse modelo existe uma norma subjetiva, definida como a percepção do individuo quanto o que as outras pessoas pensam que determinado comportamento deva ser realizado (DAVIS et al., 1989). O modelo apresenta limitações, como o risco de existir confusão entre o significado de atitudes e das normas. Outro aspecto é que se alguém apresenta uma intenção de agir, essa pessoa não necessariamente irá realizar a ação, pois existem situações como habilidade limitada, tempo, hábitos inconscientes, ou variáveis ambientais ou organizacionais que podem limitar a liberdade de agir. (BOBSIN, 2007)

Passados mais de uma década da criação da TRA, Ajzen (1991) propõe a Teoria do Comportamento Planejado (Theory of Planned Behavior - TPB), procurando complementar a TRA agregando-lhe agora controle comportamental percebido (perceived behavioral control) (DILLON; MORRIS, 1996). Ajzen (1991) entende que a intenção de comportamento se reflete no comportamento se a pessoa decidir por vontade própria adotá-lo ou não, ou seja, pelo controle percebido que ela tem sobre o comportamento que deseja. O comportamento é produto de uma série de eventos cognitivos e afetivos precedidos, muitas vezes, pela intenção consciente de agir.

Essa teoria também tem seu foco na relação entre uso e atitude do usuário e discute a existência de normas subjetivas que cercam a performance do comportamento e a percepção individual relacionada à facilidade com que um comportamento pode ser realizado. O controle do comportamento e a percepção frente à dificuldade em realizar determinado comportamento. Em virtude de identificar que existe certa dificuldade de avaliar o controle real, os autores passaram a trabalhar com o controle percebido. (VENKATESH et al., 2003)

Outro modelo proposto por Davis (1989), como uma adaptação do modelo da Teoria da Ação Racional (TRA), foi o Modelo de Aceitação de Tecnologia (Technology Acceptance Model - TAM). Segundo Davis (1989), por ser tão universal, o TRA foi modificado especificamente, para criar modelos de aceitação das tecnologias de informação, como no caso específico do TAM.

O modelo TAM foi projetado para compreender a relação causal entre variáveis externas de aceitação dos usuários e o uso real do computador, buscando entender o comportamento do usuário através do conhecimento da utilidade e da facilidade de utilização percebida por ele (DAVIS, 1989). Para Davis (1989), as pessoas tendem a usar ou não uma tecnologia com o objetivo de melhorar seu desempenho no trabalho - utilidade percebida. Porém, mesmo que a pessoa entenda que uma determinada tecnologia é útil, sua utilização poderá ser prejudicada se o uso for muito complicado, de modo que o esforço não compensa o uso - facilidade percebida. Sendo assim, o TAM está baseado basicamente em dois construtos: a utilidade percebida e a facilidade de uso percebida, sendo que ambos medeiam completamente os efeitos das variáveis externas, como características do sistema, processo de desenvolvimento, treinamento e intenção de uso (DAVIS,1989). A intenção desse modelo é representar o impacto de fatores externos relacionados ao sistema de informação, sobre aqueles internos do indivíduo, como as atitudes e intenções de uso. (DAVIS; BAGOZZI; WARSHAW, 1989; DAVIS 1989) 
Tem-se ainda o modelo de Adequação entre Tarefa e Tecnologia (Task Technology Fit - TTF), a estreita relação entre tarefa, tecnologia e desempenho individual tem sido uma preocupação constante nas pesquisas de Sistemas de Informação. Esse modelo indica que a performance do indivíduo é influenciada pelo ajuste existente entre as tarefas que ele realiza $e$ a funcionalidade da tecnologia, ou seja, quanto mais de acordo com as tarefas realizadas o sistema estiver, melhor será o desempenho do indivíduo (GOODHUE; THOMPSON, 1995). Esse modelo inclui a performance do indivíduo, apresentando que a utilização do sistema $e$, consequentemente, a performance são influenciadas pela relação existente entre as características do SI utilizado, que deve estar de acordo com as características das tarefas realizadas. (BOBSIN, 2007)

O modelo TTF foca na tarefa do usuário e na funcionalidade do SI disponível (DISHAW; STRONG, 1999), que, quando ajustados, aumentam o nível de utilização da tecnologia e melhoram o desempenho do usuário e da organização.

Analisando de forma comparativa os modelos TAM e TTF, percebe-se que o que diferencia um modelo do outro, e o foco na tarefa, pois a tecnologia é uma ferramenta para que se realizem as tarefas organizacionais. O TAM inclui, de modo implícito, a tarefa, quando avalia a utilização dos SI a partir da utilidade percebida, enquanto o modelo TTF trabalha de forma explícita as características da tarefa para avaliar a utilização dos SI. (DISHAW e STRONG, 1999)

O estudo realizado por Dishaw e Strong (1999), Klopping e McKinney (2004) avaliou o uso da tecnologia a partir da integração dos modelos TAM e TTF. Dishaw e Strong (1999) focaram na integração dos dois modelos tendo em vista o TTF incluir a tarefa, sendo esse fato considerado pelos autores uma fraqueza do modelo TAM. Ao aplicarem o modelo integrado, na análise de um software de gerenciamento de projetos, eles encontraram um percentual de explicação maior (DISHAW; STRONG, 1999) do que o percentual do modelo TAM.

O modelo integrado apresentado ajuda a compreender o porque de os indivíduos escolherem determinadas tecnologias para auxiliar em suas tarefas particulares, já que essa compreensão torna-se importante para os profissionais da área de SI, além de ajudar a entender como as características da tecnologia e o seu ajuste com as características da tarefa levam o usuário a escolher determinada ferramenta. (DISHAW; STRONG, 1999)

Segundo os autores, a combinação dos modelos poderia melhor explicar a utilização da tecnologia (DISHAW; STRONG, 1999; KLOPPING; MCKINNEY, 2004). Dessa forma, percebe-se que o uso dos sistemas de informações não está relacionado somente com o ajuste entre a tarefa e a tecnologia, ou com o comportamento $e$ as atitudes dos indivíduos frente aos SI, mas sim com a combinação destes fatores. A partir da integração dos modelos, é possível identificar o comportamento de utilização dos sistemas de informações, o qual e determinado pelas variáveis: utilidade percebida; facilidade de uso percebida; intenção de uso; e ajuste entre tarefa e tecnologia.

Diante dos inúmeros métodos apresentados, optou-se por trabalhar com variáveis que contemplam a relação entre a tecnologia e as tarefas desempenhadas pelos usuários e o comportamento e as atitudes dos indivíduos frente aos SI.

\section{Metodologia de Pesquisa}

Este artigo caracteriza-se por uma pesquisa descritiva, pois possui planos estruturados (HAIR et al., 2005a). Nesta pesquisa, se trabalhou com a integração de variáveis que abordam o comportamento e atitude dos indivíduos frente à tecnologia e ao ajuste desta com as tarefas executadas. As pesquisas já realizadas (VLAHOS, et al., 2004; GOODHUE, 1995; DAVIS, 1989; KLOPPING; MCKINNEY, 2004) auxiliaram na definição das variáveis deste estudo, visto que tal estudo pretende identificar o uso dos SI.

Conforme apresentado inicialmente, esta pesquisa pretende investigar os fatores que explicam a utilização do Gerenciador de Capacitação (GECAP) na realização das atividades dos servidores da ESAT, trabalhando com variáveis que contemplam o comportamento $e$ a atitude dos indivíduos frente à tecnologia e o ajuste destas com as tarefas executadas.

Nesse sentido, entende-se que o uso é determinado por fatores como facilidade de utilização percebida, utilidade percebida, intenção de uso do GECAP e o ajuste entre a tarefa e a tecnologia. 
Neste artigo será trabalhado com o construto ajuste tarefa-tecnologia (TTF) e não com as variáveis que caracterizam as tarefas e a tecnologia. Optou-se por trabalhar desta maneira tendo em vista o estudo de Klopping e McKinney (2004) apresentar o seu modelo com o construto ajuste, pois, para os autores, o comportamento de uso pode estar relacionado com a percepção do indivíduo em relação ao ajuste entre a tarefa e a tecnologia.

Outra observação importante quanto às variáveis que contemplam o comportamento dos indivíduos é o fato de não se trabalhar com a atitude para uso, pois Davis e Venkatesh (1996, p. 21) apresentam que a partir de "[...] evidências empíricas, o modelo final exclui o construto atitude porque este não mede completamente o efeito da percepção da utilidade na intenção [...]", visto que o uso é explicado pelos fatores determinantes, os quais, dentre tantas variáveis, contemplam as tarefas executadas pelos indivíduos. (BOBSIN, 2007)

\subsection{Operacionalização das Variáveis}

As variáveis que serão estudadas indicam que o uso do GECAP depende da aceitação do sistema, ou seja, a identificação pelo indivíduo da utilidade dele e a facilidade de manuseio; e do nível de ajuste entre a tarefa a ser realizada e o SI da empresa. Dessa forma, tem-se como variáveis independentes, as quais poderão afetar, influenciar ou determinar as demais variáveis (MARCONI; LAKATOS, 2003): facilidade de utilização percebida, utilidade percebida, atitude para o uso e ajuste entre a tarefa e a tecnologia, enquanto a intensidade de uso é variável dependente.

$\mathrm{O}$ instrumento de pesquisa analisa o uso dos SI por meio da integração de variáveis referentes ao ajuste entre tarefa, tecnologia, comportamento e atitude dos usuários. O comportamento e a atitude para uso são estudados com base em Davis e Venkatesh (1996), Dishaw e Strong (1999), Klopping e McKinney (2004) e apresentam os seguintes fatores, que estão compreendidas no questionário deste trabalho de acordo com (BOBSIN, 2007): utilidade percebida, facilidade de utilização percebida, intenção de uso, ajuste entre a tarefa e a tecnologia.

As 27 variáveis referentes aos determinantes do uso dos SI foram medidas através de escala Likert de cinco pontos que variam do discordo totalmente até o concordo totalmente.

A intensidade de uso foi medida pelo tempo e frequência de utilização da ferramenta pelo indivíduo (DISHAW; STRONG, 1999; KLOPPING; MCKINNEY, 2004; GOODHUE, 1995; VLAHOS et al., 2004). Legris et al., (2003) apresentam que o uso é medido normalmente através de duas ou três questões sobre frequência de uso, quantidade de tempo gasto usando o sistema.

Com base nos aspectos expostos, o instrumento de pesquisa, em forma de questionário, apresentou 30 variáveis, foi testado e validado na dissertação da Débora Bobsin (2007), na UFSM-RS.

Tal validação se faz necessária, pois apesar de existirem na literatura questionários com seu uso amplamente difundido, não se encontrou nenhum validado no Brasil (BOBSIN, 2007) e, segundo Oliveira Neto e Riccio (2003), a validação cultural de um questionário é essencial antes de sua aplicação, pois conceitos bem difundidos em uma cultura podem não existir em outras.

\subsection{População}

A população desta pesquisa foi concentrada nos 14 servidores públicos, independentemente da função exercida, que trabalham diretamente com o GECAP da ESAT, sediada no bairro do Distrito Industrial, na cidade de João Pessoa.

\subsection{Coleta de Dados}

Este estudo compreende uma pesquisa survey, sendo assim, a coleta de dados deu-se por meio de questionários guiados pela hipótese destacada, que foi formulada com base na teoria, e apresenta o que precisa ser medido. (HAIR et al., 2005a)

A escolha por essa forma de coleta de dados ocorreu devido a sua estruturação, certa padronização, provocando informações específicas (MALHOTRA, 2006), situação desejada neste trabalho.

A coleta ocorreu no período de 19 a 23 do mês de março de 2012. Os questionários foram entregues aos servidores da escola que responderam aos seus questionários no mesmo período, entretanto, eles foram entregues e recolhidos pelo próprio pesquisador, 
o qual já repassava as informações básicas quanto ao instrumento.

\section{Resultados da Pesquisa}

O GECAP analisado neste estudo foi desenvolvido exclusivamente para ESAT onde se realizou a pesquisa. Esse SI foi criado tendo em vista a grande perda de tempo no desenvolvimento de processos manuais, pela falta de padronização no setor de trabalho, como também, por causa do aumento das demandas administrativas, pois o número de servidores para atendê-las é muito pequeno, foram aspectos decisivos para a implantação desse SI. Assim, pode-se integrar os setores otimizando os serviços e unificando as informações.

A descrição do sistema foi efetuada com base em informações disponibilizadas pela empresa e pelos profissionais que o desenvolveram. O SI possui um banco de dados de todos os servidores da Secretaria de Estado da Receita projetado para atender às necessidades da ESAT.

O software é dividido no perfil gerencial e administrativo, em que foram distribuídos os perfis de acordo com os cargos existentes, portanto, os cargos de chefia e gerência possuem perfil de acesso mais detalhado, podendo recorrer a outros recursos da ferramenta.

\subsection{Perfil dos Respondentes}

Foram preenchidos os 14 questionários distribuídos. Ao analisar o perfil dos respondentes, verificou-se um número maior de mulheres, pois representam $71,43 \%$ dos respondentes, frente a $28,57 \%$ que correspondem aos indivíduos do sexo masculino. Quanto à idade, verifica-se um público maduro com média de 42,7 anos, evidencia-se que $85,7 \%$ dos colaboradores da empresa têm de 36 a 60 anos.

Identificou-se que três respondentes, ou seja, $21,42 \%$ possuem pós-graduação, quatro servidores, $28,56 \%$ possuem nível superior completo e outros quatro ensino médio completo. Dois servidores com ensino superior incompleto $(14,28 \%)$, um servidor apenas possui ensino médio incompleto $(7,14 \%)$.
Quanto ao tempo de empresa, verificou-se que os colaboradores estão em média há seis anos na empresa, sendo que os funcionários que têm até quatro anos de empresa correspondem a 78,58\% dos respondentes.

\subsection{Análise dos Itens dos Construtos}

As variáveis estão apresentadas na ordem do questionário, ou seja, primeiro as variáveis que se referem à utilidade percebida, seguida de facilidade de uso percebida, intenção de uso e ajuste entre tarefa e tecnologia.

Os resultados da Tabela 1 mostram que os respondentes percebem a utilidade dos sistemas de informações, visto que, para todas as variáveis a média teve resultado próximo de quatro, apresentando concordância em relação às afirmações. Dessa forma, as pessoas veem os SI como uma ferramenta que aumenta sua produtividade e adiciona valor ao trabalho. Nesse sentido, Goodhue (1995) apresenta que as organizações gastam milhões de dólares em sistemas de informações com o intuito de melhorar, tanto o desempenho organizacional, quanto o desempenho individual de seus colaboradores. Entretanto, Venkatesh et al. (2003) apresentam que a tecnologia só melhora a produtividade do usuário se ela for aceita e utilizada pelos indivíduos.

Tabela 1: Média e desvio padrão das variáveis que compõem o fator Utilidade Percebida

\begin{tabular}{|c|c|c|c|}
\hline $\begin{array}{l}\text { N. } \\
\text { ORD. }\end{array}$ & $\begin{array}{c}\text { UtiLIDADE PeRCEBIDA - VA- } \\
\text { RIÁVEL }\end{array}$ & MÉdIA & $\begin{array}{l}\text { Desvio } \\
\text { P. }\end{array}$ \\
\hline 01 & $\begin{array}{l}\text { Usar o SI permite-me realizar } \\
\text { mais rapidamente as minhas } \\
\text { tarefas. }\end{array}$ & 4,29 & 0,83 \\
\hline 02 & $\begin{array}{l}\text { Usar o sistema de informações } \\
\text { aumenta a minha } \\
\text { produtividade. }\end{array}$ & 3,86 & 0,95 \\
\hline 03 & $\begin{array}{l}\text { O SI é importante e adiciona } \\
\text { valor ao meu trabalho. }\end{array}$ & 3,86 & 0,95 \\
\hline 04 & $\begin{array}{l}\text { O sistema prejudica meu } \\
\text { desempenho no trabalho }\end{array}$ & 1,21 & 0,43 \\
\hline 05 & $\begin{array}{l}\text { Usar o SI facilita a realização } \\
\text { do meu trabalho. }\end{array}$ & 4,29 & 0,83 \\
\hline 06 & $\begin{array}{l}\text { O sistema de informações é útil } \\
\text { para as minhas tarefas. }\end{array}$ & 4,21 & 0,8 \\
\hline
\end{tabular}

Fonte: Dados da pesquisa 
As variáveis "Usar o SI permite-me realizar mais rapidamente as minhas tarefas" e "Usar o SI facilita a realização do meu trabalho" foram as que apresentaram as maiores médias, no entanto, o menor desvio padrão $(0,43)$ se deu na variável "o sistema prejudica meu desempenho no trabalho", mesmo tendo uma média muito baixa, logo se subentende que o SI não prejudica o desempenho no trabalho. Verifica-se que o desvio-padrão de todas as questões teve resultado menor do que um, apresentando pouca dispersão dos respondentes quanto à concordância em relação às afirmações.

Além desses aspectos, os indivíduos também concordaram que gostam de usar os sistemas de informações em suas tarefas.

Esses resultados corroboram os dados da pesquisa de Silva (2005) e Bobsin (2007), quando os respondentes também concordaram que os SI aumentam sua produtividade, permitindo terminar as tarefas mais rapidamente, considerando uteis ao trabalho, além de apresentarem que eles gostam de usar os SI em suas tarefas.

Tabela 2: Media e desvio padrão das variáveis que compõem o fator Facilidade de Uso Percebida

\begin{tabular}{|c|c|c|c|}
\hline $\begin{array}{l}\text { N. } \\
\text { ORD. }\end{array}$ & $\begin{array}{c}\text { Facilidade de Uso Percebida - } \\
\text { VARIÁvel }\end{array}$ & MÉdiA & $\begin{array}{l}\text { Desvio } \\
\text { P. }\end{array}$ \\
\hline 01 & $\begin{array}{l}\text { Aprender a utilizar/operar o SI foi } \\
\text { difícil para mim. }\end{array}$ & 1,79 & 0,7 \\
\hline 02 & $\begin{array}{l}\text { Foi necessário muito tempo para } \\
\text { eu aprender a utilizar/operar o } \\
\text { SI. }\end{array}$ & 1,71 & 0,61 \\
\hline 03 & $\begin{array}{l}\text { Eu frequentemente me confundo } \\
\text { ao utilizar o sistema. }\end{array}$ & 1,71 & 0,47 \\
\hline 04 & $\begin{array}{l}\text { Utilizar/operar o GECAP permite } \\
\text { me tornar mais habilidoso. }\end{array}$ & 3,36 & 1,01 \\
\hline 05 & $\begin{array}{l}\text { A interação com o GECAP não } \\
\text { me exige muito esforço mental. }\end{array}$ & 3,64 & 1,34 \\
\hline 06 & $\begin{array}{l}\text { Eu considero o sistema de } \\
\text { informações fácil de usar. }\end{array}$ & 4,21 & 0,58 \\
\hline
\end{tabular}

Fonte: Dados da pesquisa

Observando os itens "aprender a utilizar/operar o SI foi difícil para mim", "foi necessário muito tempo para eu aprender a utilizar/operar o SI" e "eu frequentemente me confundo ao utilizar o sistema", percebe-se que os respondentes discordaram das afirmações, conforme os resultados obtidos nas médias das variáveis.

As questões "utilizar/operar o GECAP permite me tornar mais habilidoso", e "a interação com o GECAP não me exige muito esforço mental" foram retiradas do questionário Bobsin (2007), pois obtiveram no teste de validação correlações de valor menores do que 0,6 , quando através do teste das correlações que se mostraram significativas a partir do teste de Kaise-Meyer-Olkin (KMO) de valor 0,933, o qual deve ser maior ou igual a 0,6 para que a correlação entre cada par de variáveis seja explicada pelas demais variáveis do estudo (LATIF, 2004). Isso explica também o fato de seu alto desvio padrão, apresentando-se com valor maior que um, mostrando uma alta dispersão das respostas dessas variáveis.

As variáveis do fator "facilidade de uso percebida" afirmam quanto o usuário considera que o SI é de fácil operação. Ferreira e Leite (2003) mostram que os SI devem possuir a característica de usabilidade, a qual determina que a tecnologia é de fácil manuseio $e$ rápido aprendizado. $\mathrm{O}$ que faz com que se resolvam facilmente as tarefas para as quais a ferramenta foi projetada. Dessa forma, essas características fazem com que o SI seja bem aceito e, consequentemente, utilizado pelos indivíduos na realização de suas tarefas.

Os servidores consideram o SI fácil de usar, visto que a média para esta questão foi de 4,21. Isso denota que os requisitos não funcionais, dentre eles os de interface, indicam a qualidade do sistema, ou seja, suas facilidades (FERREIRA; LEITE, 2003). Para os autores, esses requisitos se relacionam com a exibição da informação e a entrada de dados.

De acordo com Mathieson e Keil (1998), a não percepção da facilidade de uso pode ocorrer em situações nas quais as tarefas em que o SI está preparado para dar suporte não correspondem às tarefas que os indivíduos esperam que a ferramenta os auxilie a executar. Os autores, ainda, afirmam que existe relação entre a facilidade de uso percebida e o ajuste entre a tarefa e a tecnologia. 
Tabela 3: Media e desvio padrão das variáveis que compõem o fator Intenção de Uso

\begin{tabular}{|c|c|c|c|}
\hline $\begin{array}{c}\text { N. } \\
\text { ORD. }\end{array}$ & INTENÇÃO DE Uso - VARIÁVEL & MÉdia & $\begin{array}{c}\text { Desvio } \\
\text { P. }\end{array}$ \\
\hline 01 & $\begin{array}{l}\text { Eu acredito que é muito bom } \\
\text { usar o SI nas minhas tarefas, ao } \\
\text { invés de métodos manuais. }\end{array}$ & 4,64 & 0,63 \\
\hline 02 & $\begin{array}{l}\text { Eu desejo usar o GECAP } \\
\text { para as minhas tarefas em } \\
\text { complementação aos métodos } \\
\text { manuais. }\end{array}$ & 3,14 & 1,56 \\
\hline 03 & $\begin{array}{l}\text { É muito melhor para mim, usar } \\
\text { o SI na realização das minhas } \\
\text { tarefas ao invés dos métodos } \\
\text { manuais. }\end{array}$ & 4,29 & 0,83 \\
\hline 04 & $\begin{array}{l}\text { Eu gosto de usar o GECAP para } \\
\text { as minhas tarefas. }\end{array}$ & 3,86 & 0,95 \\
\hline 05 & $\begin{array}{l}\text { Minha intenção é utilizar o SI ao } \\
\text { invés de métodos manuais para } \\
\text { executar as minhas tarefas. }\end{array}$ & 3,93 & 0,83 \\
\hline
\end{tabular}

Fonte: Dados da pesquisa

Verifica-se que os resultados dessas variáveis concordam que o uso dos SI para as suas tarefas se sobressai em relação ao uso de métodos manuais. Entretanto, examina-se que os respondentes estão mais propensos a concordar com o fato de ser bom usar o SI, e não que isso seja muito melhor do que as ferramentas manuais. Isso se confere pelo fato de a variável "eu acredito que é muito bom usar o SI nas minhas tarefas, ao invés de métodos manuais" apresentar a maior média, ou seja, maior indicação de concordância e menor desvio padrão, o que indica pouca dispersão nas respostas, em relação às demais variáveis do construto Bobsin (2007).

As questões "eu desejo em usar o GECAP para as minhas tarefas em complementação aos métodos manuais" e "eu gosto de usar o GECAP para as minhas tarefas" foram retiradas do questionário Bobsin (2007) pelos mesmos motivos da facilidade de uso percebida da tabela anterior.

Outra medida relevante foi "minha intenção é utilizar o SI ao invés de métodos manuais para executar as minhas tarefas". Para Davis et al.. (1989), a intenção de uso é o principal determinante do comportamento de uso dos SI. Wu et al. (2004) apresentam que a utilidade percebida e a facilidade de uso percebida explicam as diferenças de intenção de uso. Segundo os autores, se a utilidade e a facilidade de uso do SI forem percebidas, o individuo desenvolverá intenção de uso e, consequentemente, utilizará a tecnologia Bobsin (2007).

Tabela 4: Media e desvio padrão das variáveis que compõem o fator Ajuste Tarefa-tecnologia

\begin{tabular}{|c|c|c|c|}
\hline $\begin{array}{l}\text { N. } \\
\text { ORD. }\end{array}$ & $\begin{array}{c}\text { AJUSTE ENTRE A TAREFA E A } \\
\text { TECNOLOGIA - VARIÁvel }\end{array}$ & MÉDIA & $\begin{array}{l}\text { Desvio } \\
\text { P. }\end{array}$ \\
\hline 01 & $\begin{array}{l}\text { Os dados são apresentados } \\
\text { em um nível de detalhamento } \\
\text { suficiente para as minhas } \\
\text { tarefas. }\end{array}$ & 3,57 & 0,65 \\
\hline 02 & $\begin{array}{l}\text { No SI, a informação é óbvia e } \\
\text { fácil de encontrar. }\end{array}$ & 4 & 0,96 \\
\hline 03 & $\begin{array}{l}\text { Quando eu necessito do SI, eu } \\
\text { fácil e rapidamente localizo a } \\
\text { informação. }\end{array}$ & 4 & 0,96 \\
\hline 04 & $\begin{array}{l}\text { As informações que utiliza ou } \\
\text { que eu gostaria de utilizar são } \\
\text { exatas o suficiente para as } \\
\text { minhas finalidades. }\end{array}$ & 3,21 & 0,89 \\
\hline 05 & $\begin{array}{l}\text { As informações são atuais } \\
\text { o suficiente para as minhas } \\
\text { finalidades. }\end{array}$ & 3,29 & 0,99 \\
\hline 06 & $\begin{array}{l}\text { As informações que eu } \\
\text { necessito são apresentadas } \\
\text { de forma que facilita a } \\
\text { compreensão. }\end{array}$ & 3,71 & 1,07 \\
\hline 07 & $\begin{array}{l}\text { A informação é armazenada em } \\
\text { diferentes formatos e é difícil } \\
\text { saber qual usar eficazmente }\end{array}$ & 2,29 & 1,38 \\
\hline 08 & $\begin{array}{l}\text { Eu facilmente encontro a } \\
\text { definição exata dos dados } \\
\text { necessários para realizar as } \\
\text { minhas tarefas. }\end{array}$ & 3,57 & 0,94 \\
\hline 09 & $\begin{array}{l}\text { Os dados que eu necessito ou } \\
\text { utilizo são confiáveis. }\end{array}$ & 3,79 & 0,7 \\
\hline 10 & $\begin{array}{l}\text { Eu facilmente consigo agregar } \\
\text { dados ao SI ou comparar } \\
\text { dados. }\end{array}$ & 3,64 & 1,08 \\
\hline
\end{tabular}

Fonte: Dados da pesquisa

As variáveis que compõem o construto "ajuste entre a tarefa e a tecnologia" apresentaram médias menores do que as demais variáveis. Entretanto, os resultados denotam que os respondentes tenderam a concordar com as afirmações.

Das dez variáveis analisadas, duas foram descartadas do questionário Bobsin (2007), pelos mesmos motivos da facilidade de uso percebida e intenção de uso das tabelas anteriores, a primeira de que a 
informação é armazenada em diferentes formatos e é difícil saber qual usar eficazmente e a segunda que pergunta se eu facilmente consigo agregar dados ao SI ou comparar dados. Elas apresentaram nessa pesquisa um desvio-padrão muito alto, demonstrando a alta dispersão das informações.

Como surpresa observa-se o resultado da variável "as informações que eu necessito são apresentadas de forma que facilita a compreensão" apresentou também um desvio-padrão maior do que um, demonstrando a alta dispersão dessa variável.

Os resultados das variáveis que compõem o fator "ajuste tarefa-tecnologia" apresentaram em sua maioria resultados muito próximos de um, denotando certa dispersão deles.

Analisando esse construto, observa-se que o uso das informações ocorre à medida que os dados que se necessita são identificados, adquiridos e interpretados (GOODHUE, 1995). Conforme discutido na revisão de literatura, os SI são responsáveis por disponibilizar as informações a seus usuários. Dessa forma, entende-se o motivo pelo qual Goodhue (1995) apresenta que os usuários necessitam de dados confiáveis, em um nível de detalhamento suficiente, não podendo ter dificuldade de localizar os dados.

Tabela 5: Média e desvio padrão apenas dos dados considerados válidos por Bobsin (2007)

\begin{tabular}{|c|c|c|c|}
\hline $\begin{array}{l}\text { N. } \\
\text { ORD. }\end{array}$ & $\begin{array}{c}\text { Ajuste ENTRE A TAREFA E A } \\
\text { TECNOLOGIA - VARIÁVEL }\end{array}$ & Média & $\begin{array}{l}\text { Desvio } \\
\text { P. }\end{array}$ \\
\hline 01 & Utilidade percebida & 4,1 & 0,87 \\
\hline 02 & Facilidade de uso percebida & 4,2 & 0,6 \\
\hline 03 & Intenção de uso & 4,2 & 0,7 \\
\hline 04 & Ajuste entre tarefa e tecnologia & 3,6 & 0,9 \\
\hline
\end{tabular}

Fonte: Dados da pesquisa

O construto facilidade de uso percebida e intenção de uso apresentaram as maiores médias $(4,2)$, seguido do fator utilidade percebida $(4,1)$. O construto facilidade de uso percebida apresentou o menor desvio-padrão. Ressalta-se que esses fatores em conjunto apresentaram resultados de concordância dos indivíduos em relação às variáveis apresentadas, entretanto, o fator ajuste entre tarefa e tecnologia teve a menor média, 3,6, tendendo aos indivíduos não concordarem e nem discordarem com a ideia de que o SI esteja ajustado às suas tarefas, corroborando com os resultados obtidos por Bobsin (2007).

\subsection{Análise do Uso dos Sistemas de Informações}

O uso foi medido por meio da média de horas por semana em que os indivíduos utilizam os SI para desempenhar suas tarefas, e pela frequência de uso dos SI Bobsin (2007).

Ao se questionar as horas por semana que realmente utilizam o sistema de informações para desempenhar as suas tarefas (Tabela 6), os respondentes apresentaram uma média de quase 2,8 horas, sendo que o maior número de observações $(64,29 \%)$ encontra-se no intervalo entre 0 e 10 horas. Dos pesquisados, $37,71 \%$ usam os SI entre 31 e 40 horas por semana.

Tabela 6: Frequência das respostas da questão: Indique em média quantas horas por semana você realmente utiliza o sistema de informações para desempenhar suas tarefas

\begin{tabular}{|c|c|c|c|c|}
\hline $\begin{array}{c}\text { N. } \\
\text { ORD. }\end{array}$ & $\begin{array}{c}\text { Tempo } \\
\text { de Uso }\end{array}$ & $\begin{array}{c}\text { Fre- } \\
\text { QUÊN- } \\
\text { CIA }\end{array}$ & Porcentagem & $\begin{array}{c}\text { Porcentagem } \\
\text { Acumulada }\end{array}$ \\
\hline 1 & $\begin{array}{l}\text { De zero } \\
\text { até } 10 \\
\text { horas }\end{array}$ & 9 & $\begin{array}{c}9 \times 100 / 14= \\
64,29 \%\end{array}$ & $64,29 \%$ \\
\hline 2 & $\begin{array}{l}\text { De } 11 \text { a } \\
20 \text { horas }\end{array}$ & 0 & 0 & $64,29 \%$ \\
\hline 3 & $\begin{array}{l}\text { De } 21 \text { a } \\
30 \text { horas }\end{array}$ & 0 & 0 & $64,29 \%$ \\
\hline 4 & $\begin{array}{l}\text { De } 31 \text { a } \\
40 \text { horas }\end{array}$ & 5 & $\begin{array}{c}5 \times 100 / 14= \\
37,71 \%\end{array}$ & $100 \%$ \\
\hline \multirow[t]{4}{*}{5} & $\begin{array}{l}\text { De } 41 \text { a } \\
50 \text { horas }\end{array}$ & 0 & 0 & $100 \%$ \\
\hline & total & 14 & 100 & \\
\hline & média & 2,8 & & \\
\hline & $\begin{array}{l}\text { desvio } \\
\text { padrão }\end{array}$ & 4,1 & & \\
\hline
\end{tabular}

Fonte: Dados da pesquisa

Percebe-se uma variação muito grande na média de horas por semana que utilizam o SI, como também, períodos em que os servidores da ESAT não se enquadraram. Lembrando que não foi considerada para esta análise nenhuma classificação dos servidores através de seus cargos. 
Quanto à frequência de uso, apresentada na Tabela 12, percebe-se que $64,29 \%$ usam os SI todos os dias. Ressalta-se que o respondente que marcou a alternativa "outros" informou que não usa o GECAP.

Tabela 7: Frequência das respostas da questão: Qual a frequência com que você utiliza o SI?

\begin{tabular}{|c|c|c|c|c|}
\hline $\begin{array}{c}\text { N. } \\
\text { ORD. }\end{array}$ & $\begin{array}{c}\text { Tempo } \\
\text { de Uso }\end{array}$ & $\begin{array}{c}\text { FrE- } \\
\text { QUÊN- } \\
\text { CIA }\end{array}$ & $\begin{array}{c}\text { Porcenta- } \\
\text { GEM }\end{array}$ & $\begin{array}{l}\text { PORCENTA- } \\
\text { GEM ACU- } \\
\text { MULADA }\end{array}$ \\
\hline 1 & Todos os dias & 9 & $\begin{array}{c}9 \times 100 / 14= \\
64,29\end{array}$ & 64,29 \\
\hline 2 & $\begin{array}{l}\text { Mais de dois } \\
\text { dias na semana }\end{array}$ & 1 & $\begin{array}{c}1 \times 100 / 14= \\
7,14\end{array}$ & 71,43 \\
\hline 3 & $\begin{array}{l}\text { Menos de dois } \\
\text { dias na semana }\end{array}$ & 1 & $\begin{array}{c}1 \times 100 / 14= \\
7,14\end{array}$ & 78,57 \\
\hline 4 & Semanalmente & 1 & $\begin{array}{c}1 \times 100 / 14= \\
7,14\end{array}$ & 85,71 \\
\hline 5 & Mensalmente & 1 & $\begin{array}{c}1 \times 100 / 14= \\
7,14\end{array}$ & 92,85 \\
\hline \multirow[t]{3}{*}{6} & Outros & 1 & $\begin{array}{c}1 \times 100 / 14= \\
7,14\end{array}$ & $\begin{array}{l}99,99 \\
=100\end{array}$ \\
\hline & total & 14 & 100 & \\
\hline & desvio padrão & 4,1 & & \\
\hline
\end{tabular}

Fonte: Dados da pesquisa

\section{Considerações Finais}

Chega-se ao século XXI com um cenário organizacional bastante peculiar, pois as empresas públicas agora pretendem, cada vez mais, ser eficientes e eficazes em suas gestões e buscam por meio de seus colaboradores sua perpetuação investindo em seu capital intelectual. (BRUNI; TURRIONI; STANO, 2005)

Com relação aos SI, tem-se o desafio de conseguir construir mecanismos padronizados, integrados e interconectados que possam suportar toda essa gama de informações em seus diferentes bancos de dados e, principalmente, a disposição em trabalhar de modo a reformular, se necessário, os processos decisórios e de trabalho, compartilhando informações e os modos de oferta dos serviços, visando à adequação às necessidades e interesses dos usuários de serviços públicos.

Nesse sentido, este estudo relacionou o uso dos sistemas de informações com os servidores da ESAT, visto que os SI apresentam diferentes funções de acordo com a atividade executada pelo seu usuário, além do fato de que as tarefas com o passar do tempo vão se ajustando as necessidades de nossos usuários.

Para entender o uso do GECAP, objetiva-se analisar a percepção dos servidores públicos da Escola de Administração Tributária (ESAT) quanto ao uso do GECAP. Para isso, analisou-se em paralelo se o uso desse SI é determinado pelo comportamento e pela atitude dos indivíduos diante da tecnologia e pelo alinhamento dessa tecnologia com as tarefas executadas pelos seus usuários.

O estudo foi realizado na ESAT, onde existe um SI que auxilia a execução das tarefas da escola. A coleta de dados efetivou-se através de questionário, o qual foi validado estatisticamente antes de sua aplicação, alcançando-se, assim, um dos objetivos do estudo Bobsin (2007).

Verificou-se nesse estudo que as informações que tratam da utilidade percebida corroboram com os dados da pesquisa de Silva (2005) e de Bobsin (2007), na qual os respondentes concordaram que os SI aumentam sua produtividade, permitindo terminar as tarefas mais rapidamente, considerando-as úteis ao trabalho, além de afirmarem que gostam de usar os SI em suas tarefas.

Quanto à facilidade de uso percebida, os servidores consideraram o GECAP fácil de usar, visto que a média para essa questão foi bem alta. Percebeu-se que os respondentes estão mais propensos a concordar que seja bom usar o GECAP e não que ele seja muito melhor do que as ferramentas manuais.

Já no fator Ajuste Tarefa-Tecnologia, observou-se certa dispersão das variáveis em comparação às outras tabelas analisadas, merecendo no futuro novas análises mais detalhadas para que se percebam melhor quais interferências estão atuando na utilização da tecnologia. Esse fator (tarefa e tecnologia) teve a menor média, tendendo aos indivíduos a não concordarem e nem discordarem com a ideia de que o SI está ajustado as suas tarefas.

Observou-se que os respondentes apresentaram uma média de quase três horas na utilização do GECAP por semana, sendo que o maior número de observações encontra-se no intervalo entre 0 e 10 horas. Dessa forma, percebe-se que o uso do GECAP não se efetiva de forma padronizada dentro da ESAT, se tornando 
uma limitação desta pesquisa. Outras limitações foram identificadas, a primeira o número muito pequeno de servidores, pois com um número maior de colaboradores podería-se avaliar melhor essas variáveis. Outra limitação percebida foi a provável inibição dos respondentes, pois no questionário foi destacado o cargo que eles exerciam, podendo essa informação interferir nos resultados da pesquisa.

O fato do ajuste entre a tarefa e a tecnologia ter sido analisado com base na percepção do usuário se traduz numa análise real de pesquisa por meio da observação de suas tarefas e uso dos SI. Ressalta-se, também, que apesar de se ter pesquisado todos os colaboradores da organização, o estudo analisou uma única empresa, fato este identificado também como uma limitação.

Pesquisas como essa são muito importantes para as empresas, para que se possa compreender melhor o seu uso, e possa identificar as variáveis que realmente podem incrementar o SI, fazendo com que as empresas aumentem seu potencial ganho com a aplicação da SI.

\section{REFERÊNCIAS}

ADAMS, D.; NELSON, R.; TODD, P. Perceived usefulness, easy of use, and usage of information technology: a replication. MIS Quarterly, Estados Unidos, v. 16, p. 227-247, 1992.

\section{AFONSO, A. P. Comunidades de aprendizagem:} um modelo para a gestão da aprendizagem. [2011]. Disponível em: <http://www.nonio.uminho.pt/ documentos/actas/actchal2001/048- Ana\%20Afonso \%20 427-432.pdf>. Acesso em: 30 ago. 2011.

\section{ALAVI, M.; JOACHIMSTHALER, E. A. Revisiting DSS}

implementation research: a meta analysis of the literature and suggestions for researchers. MIS Quarterly, Estados Unidos, v. 16, n. 1, p. 95-116, 1992.

ALMEIDA, F. C. Atores e fatores na introdução de um sistema de informação. RAC, Brasil, v. 1, n. 4, p. 177192, set. 1995.

ASSMANN, Hugo. A metamorfose do aprender na sociedade da informação. 1. ed. Petrópolis, RJ: Vozes, 2005. (124p.)
BRUNI, Márcia; TURRIONI, João Batista; STANO, Rita de Cássia. Abordagens da aprendizagem no contexto organizacional. II Simpósio de Excelência em Gestão e Tecnologia - SEGeT, 2005. Disponível em: <http://www. aedb.br/seget/artigos05/274_Abordagens\%20da\%20 aprendizagem \%20no\%20contexto\%20organizacional. pdf>. Acesso: 13 fev. 2012.

BAILEY, J. E.; PEARSON, S. Development of a tool for measuring and analyzing computer user satisfaction.

Management Science, Estado Unidos, v. 29, n. 5, p. 530-545, 1983.

BALLANTINE, J. et al. The 3D model of information systems success: the search for dependent variable continues. Information Resources Management Journal, Estados Unidos, v. 9, n. 4, p. 5-14, 1996.

\section{BERTERO, C. O. Cultura organizacional e} instrumentalização do poder. In: FLEURY, M. T. L.; FISHER, R. M. (Coord.) Poder e cultura nas organizações. São Paulo: Atlas, 1989. (168p.)

BAROUDI, J. J.; ORLIKOWSKI, W. J. A short form measure of user information satisfaction: a psychometric evaluation and notes on use. Journal of Management Information Systems, Estados Unidos, v. 4, n. 4, p. 44-59, 1988.

BENBASAT, I.; DEXTER, A. S. Individual differences in the use of decision support Aids. Journal of accounting research, v. 20, n. 1, p. 1-11, 1982.

\section{BITENCOURT, Cláudia Cristina. Competência} gerencial e aprendizagem nas organizações. São Leopoldo: Editora Unisinos, 2005.

BOBSIN, Débora. A percepção dos diferentes níveis hierárquicos quanto ao uso de um sistema de informação. 2007, P. UFSC. Disponível em: < http:// www.ufsm.br/adm/mestrado/dissertacoes/debora_bobsin/ dissertacao_debora_bobsin.pdf $>$. Acesso em: 30 jan. 2012.

CAMPOS FILHO, M. P. Os sistemas de informações e as modernas tendências da tecnologia e dos negócios. RAE, Brasil, v. 34, n. 6, p. 33-45, nov.-dez. 1994. 
CHOE, J. The relationships among performance of accounting information systems. Influence Factors and evolution level of information systems. Journal of Management Information Systems. Estados Unidos, v. 12 , n. 4, p. 215-239, 1996.

COGHLAN, D. A person-centered approach to dealing with resistance to change. Leadership and Organization Development Journal, Bradford, v. 14, n. 4, p. 10-14, 1993.

\section{DAVIS, F. A technology acceptance model for} empirically testing new end user information systems: theory and results. [S.1.]: Thesis (Doctoral) MIT, 1986.

DELONE, W. H.; MCLEAN, E. R. Information systems success: the quest of dependent variable. Information System Research, Estados Unidos, v. 3, n. 1, p. 60-95, 1992.

\section{DIAS, D. Motivação e resistência ao uso da} tecnologia da informação: um estudo entre gerentes. RAC, Brasil, v. 4, n. 2, p. 51-66, maio-ago. 2000.

DIAS, Guilherme Ataíde; SILVA, Patrícia Maria; DELFINO, João Bosco Júnior; ALMEIDA, Josemir

Ramos. Technology Acceptance Model (TAM). [2012]. Disponível em: <http://www.brapci.ufpr .br/documento. php?dd0 $=0000011071 \& d d 1=d 3 e e a>$. Acesso em: 16 jan. 2012.

FERREIRA, Simone Bacellar Leal; LEITE, Julio Cesar Sampaio do Prado. Avaliação da usabilidade em sistemas de informação: o caso do sistema submarino. [2012]. Disponível em: < http://anpad.org.br/rac/vol_07/ dwn/rac-v7-n2-sbf.pdf> . Acesso em: 6 fev. 2012.

GONÇALVES, J. E. L. Os impactos das novas tecnologias nas empresas prestadoras de serviços. Revista de Administração de Empresas, São Paulo, v. 1, n. 34, p. 63-68, jan.-fev. 1994.

IGBARIA, M.; IIVARI, J.; MARAGAHH, H. Why do individuals use computer technology? A finnish case study. Information \& Management, v. 29, p. 227-238, 1995. Disponível em: <http:/www.sciencedirect.com/ science/article/pii/0378720695000310 > . Acesso em: 20 abr. 2012.
IGBARIA, M.; PARASURAMAN, S. A path analytic study of individual characteristics, computer anxiety and attitudes toward microcomputers. Journal of Management, v. 15, p. 373-388, 1989. Disponível em: $<$ http://jom.sagepub.com/content/15/3/373.short>. Acesso em: 20 abr. 2012.

LAKATOS, Eva Maria; MARCONI, Marina de Andrade. Fundamentos de metodologia científica. 5. ed. São Paulo: Atlas, 2003.

\section{LAUDON, Kenneth. Sistemas de Informação}

Gerenciais. São Paulo: Pearson Prentice Hall, 2010.

MATTAR, F. N. Pesquisa de marketing. São Paulo: Atlas, 1996. (v.1, 336p.)

MySQL. Why MySQL (em inglês). [2012].

Disponível em: <http://www.mysql.com/why-mysql/> . Acesso em: 28 fev. 2012.

OLIVEIRA, Antônio Flávio de. O Servidor público e a nova ordem constitucional. Revista Tribunal de Contas do Município, Rio de Janeiro, Ano XXIV, n. 37, p. 9-8, janeiro, 2008. Disponível em: <http://www.tcm. rj.gov. br/Noticias/3042/aR37TCMRJ.pdf> . Acesso em: 16 jan. 2012.

PHP (um acrônimo recursivo para PHP:

Hypertext Preprocessor). [2012]. Disponível em: <http:// php.net/manual/pt_BR/intro-whatis.php>. Acesso em: 28 fev. 2012.

RIBEIRO, Manuella Maia. O uso das tecnologias de informação e comunicação como ferramentas de combate à corrupção do Governo Federal: ComprasNet e o Portal da Transparência. $3^{\circ}$ Concurso de Monografias da CGU, Brasília, DF, 2008. (60 p.)

RODRIGUES, S. B. A Informática na organização e no trabalho. Revista de Administração de Empresas, São Paulo, v. 3, n. 29, p. 43-50, jul.-set. 1988.

SILVA, Andre Luiz Matos Rodrigues da. A influência do treinamento de usuários na aceitação de sistemas ERP em empresas no Brasil. 118f. Dissertação (Mestrado em Administração). Universidade Federal do Rio de Janeiro, Rio de Janeiro, 2005.

VERGARA, Sylvia Constant. Métodos de pesquisa em administração. São Paulo: Atlas, 2005. 